\title{
NONNEGATIVE MATRICES WITH PRESCRIBED ELEMENTARY DIVISORS*
}

\author{
RICARDO L. SOTO $^{\dagger}$ AND JAVIER CCAPA ${ }^{\dagger}$
}

\begin{abstract}
The inverse elementary divisor problem for nonnegative matrices asks for necessary and sufficient conditions for the existence of a nonnegative matrix with prescribed elementary divisors. In this work a Brauer type perturbation result is introduced. This result allows the construction, from a given a list of real or complex numbers $\Lambda=\left\{\lambda_{1}, \ldots, \lambda_{n}\right\}$, of certain structured nonnegative matrices with spectrum $\Lambda$ and with any legitimately prescribed elementary divisors.
\end{abstract}

Key words. Inverse elementary divisor problem, Nonnegative matrices.

AMS subject classifications. 15A18, 15A51.

1. Introduction. Let $A \in \mathbb{C}^{n \times n}$ and let

$$
J(A)=S^{-1} A S=\left[\begin{array}{cccc}
J_{n_{1}\left(\lambda_{1}\right)} & 0 & \ddots & 0 \\
0 & J_{n_{2}\left(\lambda_{2}\right)} & \ddots & \ddots \\
\ddots & \ddots & \ddots & 0 \\
0 & \ddots & 0 & J_{n_{k}\left(\lambda_{k}\right)}
\end{array}\right]
$$

be the Jordan canonical form of $A$ (hereafter $J C F$ of $A$ ). The $n_{i} \times n_{i}$ submatrices

$$
J_{n_{i}}\left(\lambda_{i}\right)=\left[\begin{array}{cccc}
\lambda_{i} & 1 & & \\
& \lambda_{i} & \ddots & \\
& & \ddots & 1 \\
& & & \lambda_{i}
\end{array}\right], i=1,2, \ldots, k
$$

are called the Jordan blocks of $J(A)$. Then the elementary divisors of $A$ are the polynomials $\left(\lambda-\lambda_{i}\right)^{n_{i}}$, that is, the characteristic polynomials of $J_{n_{i}}\left(\lambda_{i}\right), i=1, \ldots, k$. The inverse elementary divisor problem $(I E D P)$ is the problem of determining necessary and sufficient conditions under which the polynomials

*Received by the editors August 31, 2007. Accepted for publication June 2, 2008. Handling Editor: Michael Neumann.

${ }^{\dagger}$ Departamento de Matemáticas, Universidad Católica del Norte, Antofagasta, Casilla 1280, Chile (rsoto@ucn.cl, jccapa@ucn.cl). Supported by Fondecyt 1050026, Fondecyt 1085125, and Mecesup UCN0202, Chile. Part of this work was done while the authors were visiting at UC3M, Madrid, Spain. 
$\left(\lambda-\lambda_{1}\right)^{n_{1}},\left(\lambda-\lambda_{2}\right)^{n_{2}}, \ldots,\left(\lambda-\lambda_{k}\right)^{n_{k}}, n_{1}+\cdots+n_{k}=n$, are the elementary divisors of an $n \times n$ matrix $A$. It is clear that for any arbitrarily prescribed Jordan form $J$ and for any nonsingular matrix $S$, there exists a matrix $A=S J S^{-1}$ with $J$ as its $J C F$. In order that the problem be meaningful, the matrix $A$ is required to have a particular structure. When $A$ is required to be an entrywise nonnegative matrix, the problem is called the nonnegative inverse elementary divisor problem (NIEDP) (see [9], [10], [11]).

The NIEDP contains the nonnegative inverse eigenvalue problem (NIEP), which asks for necessary and sufficient conditions for the existence of an entrywise nonnegative matrix $A$ with prescribed spectrum $\Lambda$. Both problems remain unsolved. The nonnegative inverse eigenvalue problem has attracted the attention of many authors (see [1], [3], [5], [8], [12]-[16] and references therein). In contrast, only a few works are known about the NIEDP ( [6], [7], [9], [10]). In [10], Minc studied the problem for doubly quasi-stochastic matrices and gives conditions for the existence of doubly quasi-stochastic and doubly stochastic matrices with prescribed elementary divisors. These results are extended in [9] to matrices with complex eigenvalues. In [6], London studied the question of the existence of a doubly quasi-stochastic matrix having prescribed elementary divisors and diagonal elements.

In Section 2 of this paper, we consider the notation that will be used and a number of basic results related with the IEDP. In Section 3, we introduce a Brauer type perturbation result, which allows us to construct, from a given list of real or complex numbers $\Lambda=\left\{\lambda_{1}, \ldots, \lambda_{n}\right\}$, certain nonnegative matrices with constant row sums and spectrum $\Lambda$ and with any legitimately prescribed elementary divisors (that is, their product is a polynomial whose zeroes are the numbers $\lambda_{i}$ ). In particular we completely solve the NIEDP for lists of real numbers $\lambda_{1}>\lambda_{2} \geq \cdots \geq \lambda_{n} \geq 0$ and lists with $\lambda_{i}<0, i=2, \ldots, n$. By using results from the NIEP we also give sufficient conditions for the general case. The proofs are constructive, in the sense that we can always construct a solution matrix. Two examples are given in Section 4 .

2. Preliminaries. $\sigma(A)$ will denote the set of eigenvalues of $A$. We shall consider that in the $J C F$ of a matrix $A$, equal eigenvalues are consecutive.

A matrix $A=\left(a_{i j}\right)_{i, j=1}^{n}$ is said to have constant row sums if all its rows sum up to the same constant, say $\alpha$, i.e.

$$
\sum_{j=1}^{n} a_{i j}=\alpha, \quad i=1, \ldots, n .
$$

The set of all matrices with constant row sums equal to $\alpha$ is denoted by $\mathcal{C S}_{\alpha}$. It is clear that any matrix in $\mathcal{C S}_{\alpha}$ has the eigenvector $\mathbf{e}=(1,1, \ldots 1)^{T}$ corresponding to the eigenvalue $\alpha$. Denote by $\mathbf{e}_{k}$ the vector with one in the $k$-th position and zeros 
elsewhere.

Johnson [4] proved that if $\Lambda=\left\{\lambda_{1}, \lambda_{2}, \ldots, \lambda_{n}\right\}$ is the spectrum of an $n \times n$ nonnegative matrix, then $\Lambda$ is also the spectrum of a nonnegative matrix with constant row sums equal to its Perron root. This fact leads us to consider the NIEDP for matrices in $\mathcal{C S}_{\lambda_{1}}$.

A matrix $A$ is called quasi-stochastic if $A \in \mathcal{C S}_{1}$ and it is called doubly quasistochastic if $A$ and $A^{T}$ are in $\mathcal{C} \mathcal{S}_{1}$. A nonnegative quasi-stochastic matrix is called stochastic, and a nonnegative doubly quasi-stochastic matrix is called doubly stochastic. In other words, a nonnegative matrix $A$ is stochastic if and only if $A \mathbf{e}=\mathbf{e}$, and it is doubly stochastic if and only if $A J_{n}=J_{n} A=J_{n}$, where $J_{n}$ is the matrix whose entries are all $\frac{1}{n}$.

Let $S$ a nonsingular matrix such that $S^{-1} A S=J(A)$ is the $J C F$ of $A$. If $A \in$ $\mathcal{C} \mathcal{S}_{\lambda_{1}}$, then $S$ can be chosen so that $S \mathbf{e}_{1}=\mathbf{e}$ and it is easy to see that the rows of $S^{-1}=\left(\widehat{s}_{i j}\right)$ satisfy:

$$
\sum_{j=1}^{n} \widehat{s}_{1 j}=1 \text { and } \sum_{j=1}^{n} \widehat{s}_{i j}=0, \quad i=2, \ldots, n .
$$

If $T$ is an $n \times n$ matrix of the form

$$
T=\left[\begin{array}{cccc}
\lambda_{1} & * & \cdots & * \\
0 & * & \ddots & \ddots \\
\vdots & \ddots & \ddots & \ddots \\
0 & * & \cdots & *
\end{array}\right] \text { and } S=\left[\begin{array}{cccc}
1 & s_{12} & \cdots & s_{1 n} \\
1 & s_{22} & \cdots & s_{2 n} \\
\vdots & \vdots & \ddots & \vdots \\
1 & s_{n 2} & \cdots & s_{n n}
\end{array}\right]
$$

is nonsingular, then $S T S^{-1} \mathbf{e}=\lambda_{1} \mathbf{e}$. That is, $S T S^{-1} \in \mathcal{C} \mathcal{S}_{\lambda_{1}}$.

We shall denote by $E_{i j}$ the $n \times n$ matrix with 1 in the $(i, j)$ position and zeros elsewhere. The following simple perturbation allows us to change the eigenvalue associated to a Jordan block, without changing any of the remaining Jordan blocks: Let $A \in \mathbb{C}^{n \times n}$ with elementary divisors

$$
\left(\lambda-\lambda_{1}\right)^{n_{1}},\left(\lambda-\lambda_{2}\right)^{n_{2}}, \ldots,\left(\lambda-\lambda_{k}\right)^{n_{k}}, \quad n_{1}+\cdots+n_{k}=n,
$$

and let $S$ be a nonsingular matrix such that

$$
S^{-1} A S=J(A)=\operatorname{diag}\left\{J_{n_{1}}\left(\lambda_{1}\right), \ldots, J_{n_{p}}\left(\lambda_{p}\right), \ldots, J_{n_{k}}\left(\lambda_{k}\right)\right\}
$$

is the JCF of $A$. Let $\xi=\sum_{j=1}^{p-1} n_{j}, 1 \leq p \leq k$, with $\xi=0$ if $p=1$. Let $K=$ $\left\{\xi+1, \xi+2, \ldots, \xi+n_{p}\right\}$ and $C=\sum_{i \in K} E_{i, i}$. Then, for any $\alpha \in \mathbb{C}$, the matrix $A+\alpha S C S^{-1}$ 
has elementary divisors

$$
\left(\lambda-\lambda_{1}\right)^{n_{1}}, \ldots,\left(\lambda-\lambda_{p-1}\right)^{n_{p-1}},\left(\lambda-\lambda_{p}-\alpha\right)^{n_{p}},\left(\lambda-\lambda_{p+1}\right)^{n_{p+1}}, \ldots,\left(\lambda-\lambda_{k}\right)^{n_{k}} .
$$

Observe that if $A \in \mathcal{C S}_{\lambda_{1}}, C=\sum_{i \in K} E_{i, i}$ and $p=1$, then $A+\alpha S C S^{-1}$ belongs to $\mathcal{C S}_{\lambda_{1}+\alpha}$; otherwise if $1<p \leq k, A+\alpha S C S^{-1}$ belongs to $\mathcal{C} \mathcal{S}_{\lambda_{1}}$. If $A$ is doubly quasi-stochastic and $p \neq 1$, so is $A+\alpha S C S^{-1}$.

The above perturbation can be simultaneously extended to $r$ blocks in $J(A)$, $1 \leq r \leq k$. In particular for the $r$ first blocks we have

$$
C=\alpha_{1} \sum_{i=1}^{n_{1}} E_{i i}+\cdots+\alpha_{r} \sum_{i=\xi+1}^{\xi+n_{r}} E_{i i},
$$

where $\xi=\sum_{j=1}^{r-1} n_{j}$. Then $A+S C S^{-1}$ has elementary divisors

$$
\left(\lambda-\lambda_{1}-\alpha_{1}\right)^{n_{1}}, \ldots,\left(\lambda-\lambda_{r}-\alpha_{r}\right)^{n_{r}},\left(\lambda-\lambda_{r+1}\right)^{n_{r+1}}, \ldots,\left(\lambda-\lambda_{k}\right)^{n_{k}} .
$$

The following two perturbations allow us, respectively, to split out a Jordan block $J_{n_{i}}\left(\lambda_{i}\right), n_{i} \geq 2$, into Jordan blocks of smaller size and to join two or more Jordan blocks corresponding to a same eigenvalue $\lambda_{p}$ to obtain one Jordan block of a bigger size.

Let the elementary divisor $\left(\lambda-\lambda_{p}\right)^{n_{p}}$ (Jordan block $\left.J_{n_{p}}\left(\lambda_{p}\right)\right), n_{p} \geq 2$, be given. Then by using the perturbation $J(A)+C$, where

$$
\begin{aligned}
& C=-\sum_{i \in K} E_{i, i+1}, \quad \text { with } \\
& K=\left\{t_{1}, t_{2}, \ldots, t_{r}\right\} \subset\left\{\xi+1, \xi+2, \ldots, \xi+n_{p}-1\right\}, \\
& t_{1}<t_{2}<\cdots<t_{r}, r \leq n_{p}-1,
\end{aligned}
$$

we obtain the $r+1$ elementary divisors

$$
\left(\lambda-\lambda_{p}\right)^{q_{1}}, \ldots,\left(\lambda-\lambda_{p}\right)^{q_{r+1}}, \quad q_{1}+\cdots+q_{r+1}=n_{p}, \quad 1 \leq p \leq k .
$$

Now, let the elementary divisors $\left(\lambda-\lambda_{p}\right)^{m_{1}}, \ldots,\left(\lambda-\lambda_{p}\right)^{m_{q}}$ (Jordan blocks $\left.J_{m_{1}}\left(\lambda_{p}\right), \ldots, J_{m_{q}}\left(\lambda_{p}\right)\right)$ corresponding to $\lambda_{p}$. Then by using the perturbation $J(A)+C$, where

$$
\begin{aligned}
& C=\sum_{i \in K} E_{i, i+1}, \quad \text { with } \\
& K=\left\{\xi+m_{1}, \xi+m_{1}+m_{2}, \ldots, \xi+\sum_{i=1}^{q-1} m_{i}\right\}
\end{aligned}
$$


we obtain the elementary divisor of bigger size $\left(\lambda-\lambda_{p}\right)^{\gamma}, \gamma=m_{1}+\cdots+m_{q}$. We state these facts in the following lemma.

Lemma 2.1. Let $A \in \mathbb{C}^{n \times n}$ be a matrix with elementary divisors

$$
\left(\lambda-\lambda_{1}\right)^{n_{1}},\left(\lambda-\lambda_{2}\right)^{n_{2}}, \ldots,\left(\lambda-\lambda_{k}\right)^{n_{k}}, \quad n_{1}+\cdots+n_{k}=n
$$

and let $S$ be a nonsingular matrix such that $S^{-1} A S=J(A)$ is the JCF of A. Let $\xi=n_{1}+n_{2}+\cdots+n_{p-1}$.

i) If $\left(\lambda-\lambda_{p}\right)^{n_{p}}$ is a nonlinear elementary divisor, $1 \leq p \leq k$, then $A+S C S^{-1}$, where $C$ is the matrix defined in (2.3), has same spectrum as $A$ but with elementary divisors

$$
\left(\lambda-\lambda_{1}\right)^{n_{1}}, \ldots,\left(\lambda-\lambda_{p-1}\right)^{n_{p-1}},\left(\lambda-\lambda_{p+1}\right)^{n_{p+1}}, \ldots,\left(\lambda-\lambda_{k}\right)^{n_{k}}
$$

and

$$
\left(\lambda-\lambda_{p}\right)^{t_{1}-\xi},\left(\lambda-\lambda_{p}\right)^{t_{2}-t_{1}}, \ldots,\left(\lambda-\lambda_{p}\right)^{t_{r}-t_{r-1}},\left(\lambda-\lambda_{p}\right)^{\xi+n_{p}-t_{r}},
$$

ii) If $\lambda_{p}=\lambda_{p+1}=\ldots=\lambda_{p+q-1}, p+q-1 \leq k$, then $A+S C S^{-1}$, where $C$ is the matrix defined in (2.4), has same spectrum as $A$ but with elementary divisors

$$
\left(\lambda-\lambda_{1}\right)^{n_{1}}, \ldots,\left(\lambda-\lambda_{p-1}\right)^{n_{p-1}},\left(\lambda-\lambda_{p+q}\right)^{n_{p+q}}, \ldots,\left(\lambda-\lambda_{k}\right)^{n_{k}}
$$

and

$$
\begin{gathered}
\left(\lambda-\lambda_{p}\right)^{w_{1}},\left(\lambda-\lambda_{p}\right)^{w_{2}}, \ldots,\left(\lambda-\lambda_{p}\right)^{w_{q-r}}, \\
w_{1}+\cdots+w_{q-r}=n_{p}+\cdots+n_{p+q-1} .
\end{gathered}
$$

Observe that if $A \in \mathcal{C} \mathcal{S}_{\lambda_{1}}$, then $\left(A+S C S^{-1}\right) \in \mathcal{C} \mathcal{S}_{\lambda_{1}}$. If $A$ is doubly quasistochastic, so is $A+S C S^{-1}$.

REMARK 2.2. Let $C$ be the matrix

$$
C= \pm \sum_{i \in K} E_{i, i+1}, \quad K \subset\{1,2, \ldots, n-1\}
$$

and let $A$ be an $n \times n$ complex matrix with $J C F \quad J(A)=S^{-1} A S$. Then for an appropriated set $K$,

$$
\begin{aligned}
J(A)+C & =S^{-1} A S+C \\
& =S^{-1}\left(A+S C S^{-1}\right) S
\end{aligned}
$$

is the $J C F$ of $A+S C S^{-1}$. Thus, given a complex matrix $A$ with $J C F \quad J(A)=$ $S^{-1} A S$, we may obtain, trivially, a matrix $B=A+S C S^{-1}$, with $\sigma(B)=\sigma(A)$ and 
prescribed elementary divisors. Observe that if $A \in \mathcal{C S}_{\lambda_{1}}$ and $S=[\mathbf{e}|*| \cdots \mid *]$ then $\left(A+S C S^{-1}\right) \in \mathcal{C S}_{\lambda_{1}}$.

In [9, Theorem 1] Minc proves that given an $n \times n$ diagonalizable positive matrix $A$, then there exists an $n \times n$ positive matrix $B$, with $\sigma(B)=\sigma(A)$ and with arbitrarily prescribed elementary divisors, provided that elementary divisors corresponding to nonreal eigenvalues occur in conjugate pairs. As a consequence we have the following result.

TheOREM 2.3. Let $A \in \mathcal{C S}_{\lambda_{1}}$ be an $n \times n$ diagonalizable positive matrix with complex spectrum. Then there exists an $n \times n$ positive matrix $B \in \mathcal{C S}_{\lambda_{1}}$, with $\sigma(B)=$ $\sigma(A)$ and with legitimately prescribed elementary divisors (that is, their product has to be equal to the characteristic polynomial of $A$ ).

Proof. Let $S^{-1} A S=D=\operatorname{diag}\left\{\lambda_{1}, \lambda_{2}, \ldots, \lambda_{n}\right\}$ be the $J C F$ of $A$, where the eigenvalues are ordered such that $\lambda_{1}>\lambda_{2} \geq \ldots \geq \lambda_{p}$ are real and $\lambda_{p+1}, \ldots, \lambda_{n}$ are complex nonreal; equal eigenvalues are consecutive and $\overline{\lambda_{j}}=\lambda_{r+j}$ for $j=p+1, \ldots, p+$ $r$, where $r=\frac{n-p}{2}$. $S=[\mathbf{e}|*| \cdots \mid *]$ is a nonsingular matrix whose $j-t$ column is an eigenvector corresponding to $\lambda_{j}, j=1, \ldots, n$, and whose columns $j$ and $r+j$ are conjugate, $j=p+1, \ldots, p+r$. Let $C$ defined as in (2.4), with $K \subset\{2, \ldots, n-1\}$ in such a way that $D+C$ is the prescribed $J C F$. Then, as it was proved in [9, Theorem 1], $S C S^{-1}$ is real. From Remark 2.2 there exists a matrix $B=A+\epsilon S C S^{-1}, B \in \mathcal{C} \mathcal{S}_{\lambda_{1}}$, with $\sigma(B)=\sigma(A)$ and with the prescribed elementary divisors. Since $A$ is positive, then $B=A+\epsilon S C S^{-1}$ is positive for sufficiently small positive $\epsilon$.

Observe that if in the above Theorem, $A$ has real spectrum, then it does not need to be diagonalizable. In this case, however, we can not split out a Jordan block $J_{n_{i}}\left(\lambda_{i}\right), n_{i} \geq 2$, into blocks of smaller size, except if $A+S C S^{-1}$ is positive (that is, if $\epsilon=1)$.

In [10] Minc gives conditions for the existence of doubly quasi-stochastic and doubly stochastic matrices with prescribed elementary divisors. In the next section we give conditions for the existence of nonnegative matrices with constant row sums and prescribed elementary divisors.

3. Nonnegative IEDP. In this section we completely solve the NIEDP for $\Lambda=$ $\left\{\lambda_{1}, \lambda_{2}, \ldots, \lambda_{n}\right\}$ with $\lambda_{i} \geq 0$, and for $\Lambda=\left\{\lambda_{1}, \lambda_{2}, \ldots, \lambda_{n}\right\}$ with $\lambda_{i}<0, i=2, \ldots, n$. For $\Lambda=\left\{\lambda_{1}, \lambda_{2}, \ldots, \lambda_{n}\right\}$ with $\lambda_{1}>\lambda_{2} \geq \cdots \geq \lambda_{p} \geq 0>\lambda_{p+1} \geq \cdots \geq \lambda_{n}$, we give sufficient conditions. Moreover, in these cases, we can always construct a nonnegative matrix with spectrum $\Lambda$ and with any legitimately prescribed elementary divisors. In this construction, if necessary, we guarantee the nonnegativity by applying the below fundamental result (Lemma 3.1), a Brauer [2] type perturbation result (see [1], [14]). The Brauer result in [2] shows how to modify one single eigenvalue of a matrix, via a 
rank-one perturbation, without changing any of the remaining eigenvalues, and it has been employed with success to obtain sufficient conditions for the nonnegative inverse eigenvalue problem. We point out that Theorem 3.2 and Theorem 3.3 below contain the result in [10, Theorem 2], seen as results for nonnegative matrices.

Lemma 3.1. Let $\mathbf{q}=\left(q_{1}, \ldots, q_{n}\right)^{T}$ be an arbitrary $n$-dimensional vector and let $A \in \mathcal{C S}_{\lambda_{1}}$ with $J C F$

$$
S^{-1} A S=J(A)=\left[\begin{array}{cccc}
\lambda_{1} & 0 & \ddots & 0 \\
0 & J_{n_{2}}\left(\lambda_{2}\right) & \ddots & \ddots \\
\ddots & \ddots & \ddots & 0 \\
0 & \ddots & 0 & J_{n_{k}}\left(\lambda_{k}\right)
\end{array}\right] .
$$

Let $\lambda_{1}+\sum_{i=1}^{n} q_{i} \neq \lambda_{i}, i=2, \ldots, n$. Then the matrix $A+\mathbf{e q}^{T}$ has Jordan canonical form $J(A)+\left(\sum_{i=1}^{n} q_{i}\right) E_{11}$. In particular, if $\sum_{i=1}^{n} q_{i}=0$ then $A$ and $A+\mathbf{e q}^{T}$ are similar.

Proof. Let $S=\left[\mathbf{e}\left|\mathbf{S}^{(2)}\right| \cdots \mid \mathbf{S}^{(n)}\right]$. Then

$$
\begin{aligned}
B & =S^{-1}\left(A+\mathbf{e q}^{T}\right) S=J(A)+S^{-1} \mathbf{e q}^{T} S \\
& =J(A)+\left[\begin{array}{cccc}
\sum_{i=1}^{n} q_{i} & \mathbf{q}^{T} \mathbf{S}^{(2)} & \cdots & \mathbf{q}^{T} \mathbf{S}^{(n)} \\
0 & \cdots & \cdots & 0 \\
\vdots & \vdots & \vdots & \vdots \\
0 & \cdots & \cdots & 0
\end{array}\right] \\
& =\left[\begin{array}{cccc}
\lambda & \mathbf{q}^{T} \mathbf{S}^{(2)} & \cdots & \mathbf{q}^{T} \mathbf{S}^{(n)} \\
0 & J_{n_{2}}\left(\lambda_{2}\right) & \ddots & 0 \\
\vdots & \ddots & \ddots & \ddots \\
0 & \ddots & \ddots & J_{n_{k}}\left(\lambda_{k}\right)
\end{array}\right],
\end{aligned}
$$

where $\lambda=\lambda_{1}+\sum_{i=1}^{n} q_{i} \neq \lambda_{i}, i=2, \ldots, n$. First, we show that in the $J C F$ of $B$, $J(B)$, there exists only one Jordan block corresponding to $\lambda$ and it is of size 1 . Clearly, written entrywise,

$$
(B-\lambda I)^{m}=\left[\begin{array}{ccccc}
0 & * & * & \ddots & * \\
& \left(\lambda_{2}-\lambda\right)^{m} & \ddots & \ddots & \ddots \\
& & \ddots & \ddots & * \\
& & & \ddots & * \\
& & & & \left(\lambda_{k}-\lambda\right)^{m}
\end{array}\right]
$$


where $m=1,2, \ldots$. Since $\lambda \neq \lambda_{i}, \operatorname{rank}\left[(B-\lambda I)^{m}\right]=n-1$ and the number of Jordan blocks of size $k=1$ and $k \geq 2$, associated with $\lambda$, are, respectively, $n-2(n-$ $1)+(n-1)=1$ and $(n-1)-2(n-1)+(n-1)=0$.

Now, we show that if $\lambda_{2}, \ldots, \lambda_{k}$, are all distinct then in $J(B)$ there exists only one Jordan block corresponding to $\lambda_{i}, i=2, \ldots, k$, and it is of size $n_{i}$. Without loss of generality, we may assume that $\lambda_{i}=\lambda_{2}$. Then we have that $\left(B-\lambda_{2} I\right)^{m}$ is an upper triangular matrix with main diagonal

$$
\left(\lambda-\lambda_{2}\right)^{m}, \underbrace{0, \ldots, 0}_{n_{2} \text { times }}, \ldots \ldots, \underbrace{\left(\lambda_{k}-\lambda_{2}\right)^{m}, \ldots,\left(\lambda_{k}-\lambda_{2}\right)^{m}}_{n_{k} \text { times }} .
$$

Then

$$
\left\{\begin{array}{l}
\operatorname{rank}\left(B-\lambda_{2} I\right)^{m}=n-m, 1 \leq m \leq n_{2} \\
\operatorname{rank}\left(B-\lambda_{2} I\right)^{m}=n-n_{2}, \quad m \geq n_{2}
\end{array}\right.
$$

It is well known that if $r_{m}(\lambda)=\operatorname{rank}(A-\lambda I)^{m}, m=1,2, \ldots$, then the difference $d_{m}(\lambda)=r_{m-1}(\lambda)-r_{m}(\lambda)$ is equal to the total number of Jordan blocks of all sizes $k \geq m$ and that $d_{m}(\lambda)=0$ for all $m>n$. Hence, the number of Jordan blocks $J_{k}\left(\lambda_{2}\right)$ associated to $\lambda_{2}$ for $k \geq 1$ is

$$
d_{1}\left(\lambda_{2}\right)=r_{0}\left(\lambda_{2}\right)-r_{1}\left(\lambda_{2}\right)=n-(n-1)=1
$$

and since for $k=n_{2}$, we have $n-\left(n_{2}-1\right)-2\left(n-n_{2}\right)+\left(n-n_{2}\right)=1$, then the size of this block is $n_{2}$.

Now we consider the case in which there are $p \geq 2$ blocks corresponding to the same eigenvalue $\lambda_{i}$. We start assuming that $p=2$ with $\lambda_{2}=\lambda_{3}$, that is, $\lambda_{2}$ has one block of size $n_{2}$ and one block of size $n_{3}$ with $n_{2} \geq n_{3}$. In this case the sequence of ranks of powers of $B-\lambda_{2} I$ is

$$
\begin{aligned}
\operatorname{rank}\left[\left(B-\lambda_{2} I\right)^{m}\right] & =n-2 m, \quad m \leq n_{3} \\
\operatorname{rank}\left[\left(B-\lambda_{2} I\right)^{m}\right] & =n-\left(n_{2}+n_{3}\right), \quad m \geq n_{2} \text { and } \\
\operatorname{rank}\left[\left(B-\lambda_{2} I\right)^{n_{3}+i}\right] & =n-2 n_{3}-i ; \quad 1 \leq i \leq\left(n_{2}-n_{3}-1\right) ; \quad n_{2}-n_{3} \geq 2 .
\end{aligned}
$$

Since

$$
d_{1}\left(\lambda_{2}\right)=r_{0}\left(\lambda_{2}\right)-r_{1}\left(\lambda_{2}\right)=n-(n-2)=2,
$$

then there exists exactly two Jordan blocks corresponding to $\lambda_{2}$ and they are of size $n_{2}$ and $n_{3}$. On the other hand, the number of blocks of size $n_{3}$ corresponding to $\lambda_{2}$ is

$$
p_{n_{3}}=r_{n_{3}-1}\left(\lambda_{2}\right)-2 r_{n_{3}}\left(\lambda_{2}\right)+r_{n_{3}+1}\left(\lambda_{2}\right),
$$


that is,

$$
p_{n_{3}}=\left\{\begin{array}{c}
{\left[n-2\left(n_{3}-1\right)\right]-2\left[n-2 n_{3}\right]+\left[n-\left(2 n_{3}\right)\right]=2 \text { if } n_{3}=n_{2}} \\
{\left[n-2\left(n_{3}-1\right)\right]-2\left[n-2 n_{3}\right]+\left[n-\left(2 n_{3}+1\right)\right]=1 \text { if } n_{3}<n_{2} .}
\end{array}\right.
$$

The number of blocks of size $n_{2}$ corresponding to $\lambda_{2}$ is, for $n_{2}>n_{3}$,

$$
p_{n_{2}}=r_{n_{2}-1}\left(\lambda_{2}\right)-2 r_{n_{2}}\left(\lambda_{2}\right)+r_{n_{2}+1}\left(\lambda_{2}\right)=1 \text {. }
$$

For $p \geq 3$, the same argument shows that there exists exactly $p$ Jordan blocks $J_{n_{2}}\left(\lambda_{2}\right), \ldots, J_{n_{p+1}}\left(\lambda_{2}\right)$, corresponding to $\lambda_{2}$ and they are of size $n_{2}, \ldots, n_{p+1}$, respectively. $\square$

Next, we consider the following result due to Perfect [12]. She showed that the matrix

$$
A=P \operatorname{diag}\left\{1, \lambda_{2}, \lambda_{3}, \ldots, \lambda_{n}\right\} P^{-1},
$$

where $1>\lambda_{2} \geq \lambda_{3} \geq \cdots \geq \lambda_{n} \geq 0$ and

$$
P=\left[\begin{array}{cccccc}
1 & 1 & 1 & \cdots & \cdots & 1 \\
1 & 1 & & & 1 & -1 \\
1 & & & \cdots & -1 & 0 \\
\vdots & & 1 & \cdots & \cdots & 0 \\
\vdots & 1 & -1 & 0 & & \vdots \\
1 & -1 & 0 & 0 & \cdots & 0
\end{array}\right]
$$

is an $n \times n$ positive stochastic matrix. As a consequence, we establish here the following result.

Theorem 3.2. Let $\Lambda=\left\{1, \lambda_{2}, \ldots, \lambda_{n}\right\}$ with $1>\lambda_{2} \geq \cdots \geq \lambda_{n} \geq 0$. There exists a stochastic matrix $A$ with spectrum $\Lambda$ and arbitrarily prescribed elementary divisors $(\lambda-1),\left(\lambda-\lambda_{2}\right)^{n_{2}}, \ldots,\left(\lambda-\lambda_{k}\right)^{n_{k}}, n_{2}+\cdots+n_{k}=n-1$.

Proof. Let $D=\operatorname{diag}\left\{1, \lambda_{2}, \ldots, \lambda_{n}\right\}$ and let $P$ be the matrix in (3.1). Then $P D P^{-1}$ is a positive stochastic matrix with spectrum $\Lambda$ and linear elementary divisors $(\lambda-1), \ldots,\left(\lambda-\lambda_{n}\right)$, counting multiplicities. Let

$$
K=\left\{t_{1}, \ldots, t_{r}\right\} \subset\{2, \ldots, n-1\},
$$

$t_{1}<t_{2}<\cdots<t_{r}, r \leq n-1$, where $\left(t_{i}, t_{i}+1\right), i=1, \ldots, r$, define the positions in $D$ in which we desired to set 1 . If $C=\sum_{i \in K} E_{i, i+1}$, then $A=P D P^{-1}+\epsilon P C P^{-1}$, where $\epsilon>0$ is such that $\left(P D P^{-1}\right)_{i j}+\epsilon\left(P C P^{-1}\right)_{i j} \geq 0, i, j=1, \ldots, n$, is nonnegative, and since $D+\epsilon C$ and $D+C$ are diagonally similar (with $\operatorname{diag}\left\{1, \epsilon, \epsilon^{2}, \ldots, \epsilon^{n-1}\right\}$ ), then $A$ has 
$J C F$ equal to $D+C$. Since $P \mathbf{e}_{1}=\mathbf{e}$, the rows of $P^{-1}$ satisfy $(2.1)$ and $P C P^{-1} \mathbf{e}=\mathbf{0}$. Then $A \in \mathcal{C S}_{1}$. Thus, $A$ is stochastic with the desired elementary divisors. $\square$

If in Theorem 3.2, we take $\epsilon>0$ such that $\left(P D P^{-1}\right)_{i j}+\epsilon\left(P C P^{-1}\right)_{i j}>0$, then we obtain a positive stochastic matrix with prescribed elementary divisors.

Now we observe that if $C$ is the matrix

$$
C=\sum_{i \in K} E_{i, i+1}, \quad K \subset\{2,3, \ldots, n-1\}
$$

and

$$
S=\left[\begin{array}{cc}
1 & 0 \\
\mathbf{e} & I_{n-1}
\end{array}\right], \quad S^{-1}=\left[\begin{array}{cc}
1 & 0 \\
-\mathbf{e} & I_{n-1}
\end{array}\right],
$$

then by (2.2) $S C S^{-1} \in \mathcal{C} \mathcal{S}_{0}$ and it has the form

$$
S C S^{-1}=\sum_{i \in K}\left(-E_{i 1}+E_{i, i+1}\right)
$$

In [16] Suleimanova announced and Perfect [12] proved that if $\Lambda=\left\{\lambda_{1}, \ldots, \lambda_{n}\right\}$ satisfies $\lambda_{1}>0>\lambda_{2} \geq \cdots \geq \lambda_{n}$, then

$$
\lambda_{1}+\lambda_{2}+\cdots+\lambda_{n} \geq 0
$$

is a sufficient condition for the existence of an $n \times n$ nonnegative matrix $A$ with spectrum $\Lambda$. The condition (3.4) is also necessary.

Theorem 3.3. Let $\Lambda=\left\{\lambda_{1}, \ldots, \lambda_{n}\right\}$ with $\lambda_{1}>0>\lambda_{2} \geq \cdots \geq \lambda_{n}$. Then there exists a nonnegative matrix $A \in \mathcal{C S}_{\lambda_{1}}$, with spectrum $\Lambda$ and prescribed elementary divisors $\left(\lambda-\lambda_{1}\right),\left(\lambda-\lambda_{2}\right)^{n_{2}}, \ldots,\left(\lambda-\lambda_{k}\right)^{n_{k}}$, if and only if $\lambda_{1}+\lambda_{2}+\cdots+\lambda_{n} \geq 0$.

Proof. Let $D=\operatorname{diag}\left\{\lambda_{1}, \lambda_{2}, \ldots, \lambda_{n}\right\}$ and let $C=\sum_{i \in K} E_{i, i+1}$ with $K \subset\{2, \ldots, n-1\}$, in such a way that $D+C$ be the prescribed $J C F$. Let $S$ the matrix in (3.2). Then $S C S^{-1}$ has the form (3.3) and

$$
B=S\left(D+\lambda_{2} C\right) S^{-1}=\left[\begin{array}{cccccccc}
\lambda_{1} & 0 & & & & & \\
\vdots & \ddots & \ddots & & & & \\
\lambda_{1}-\lambda_{j}-\lambda_{2} & & \lambda_{j} & \lambda_{2} & & & \\
\lambda_{1}-\lambda_{j} & & & \lambda_{j} & \ddots & & \\
\lambda_{1}-\lambda_{j+1}-\lambda_{2} & & & & \lambda_{j+1} & \lambda_{2} & \\
\vdots & & & & & \ddots & \ddots \\
\lambda_{1}-\lambda_{n} & & & & & & \lambda_{n}
\end{array}\right]
$$


is a matrix in $\mathcal{C S}_{\lambda_{1}}$ with spectrum $\Lambda$. Observe that the matrices $D+\lambda_{2} C$ and $D+C$ are diagonally similar (with $\operatorname{diag}\left\{1, \lambda_{2}, \lambda_{2}^{2}, \ldots, \lambda_{2}^{n-1}\right\}$ ). Then the $J C F$ of $B$ is $D+C$. Now let

$$
\mathbf{q}^{T}=\left(\sum_{i=2}^{n} \lambda_{i},-\lambda_{2},-\lambda_{3}, \ldots,-\lambda_{n}\right)
$$

Then from a result of Brauer [2], the matrix $A=B+\mathbf{e q}^{T}, A \in \mathcal{C} \mathcal{S}_{\lambda_{1}}$, is nonnegative with spectrum $\Lambda=\left\{\lambda_{1}, \ldots, \lambda_{n}\right\}, \lambda_{i}<0, i=2, \ldots, n$, if and only if $\lambda_{1}+\cdots+\lambda_{n} \geq 0$ (see [14] for a proof of this fact) and from Lemma 3.1 it has the prescribed elementary divisors $\left(\lambda-\lambda_{1}\right),\left(\lambda-\lambda_{2}\right)^{n_{2}}, \ldots,\left(\lambda-\lambda_{k}\right)^{n_{k}}$.

In ([1], Theorem 3.3) the authors give a complex version of the SuleimanovaPerfect result: if $\left\{\lambda_{2}, \ldots, \lambda_{n}\right\} \subset\{z \in \mathbb{C}: \operatorname{Re} z \leq 0,|\operatorname{Re} z| \geq|\operatorname{Im} z|\}$, then $\Lambda=$ $\left\{\lambda_{1}, \lambda_{2}, \ldots, \lambda_{n}\right\}$ is the spectrum of an $n \times n$ nonnegative matrix $A \in \mathcal{C S}_{\lambda_{1}}$ if and only if $\lambda_{1}+\lambda_{2}+\cdots+\lambda_{n} \geq 0$. This result allows us to extend Theorem 3.3 to a complex spectrum as follows.

THEOREM 3.4. Let $\Lambda=\left\{\lambda_{1}, \lambda_{2}, \ldots, \lambda_{n}\right\}$ be a list of complex numbers satisfying $\operatorname{Re} \lambda_{i}<0$ and $\left|R e \lambda_{i}\right| \geq\left|\operatorname{Im} \lambda_{i}\right|$ for $i=2, \ldots, n$. Then if $\lambda_{1}+\cdots+\lambda_{n}>0$, there exists a nonnegative matrix $A \in \mathcal{C S}_{\lambda_{1}}$, with spectrum $\Lambda$ and with prescribed elementary divisors $\left(\lambda-\lambda_{1}\right),\left(\lambda-\lambda_{2}\right)^{n_{2}}, \ldots,\left(\lambda-\lambda_{k}\right)^{n_{k}}$.

Proof. Let $\lambda_{1}>0>\lambda_{2} \geq \cdots \geq \lambda_{p}$ be real and let $\lambda_{p+1}, \ldots, \lambda_{n}$ be complex nonreal, where $\lambda_{i}$ is conjugate to $\lambda_{i+1}$ for $i=p+1, p+3, \ldots, n-1$. Let

$$
J=\left[\begin{array}{ccccccc}
\lambda_{1} & & & & & & \\
& \ddots & & & & & \\
& & \lambda_{p} & & & & \\
& & & \operatorname{Re} \lambda_{p+1} & -\operatorname{Im} \lambda_{p+1} & & \\
& & \operatorname{Im} \lambda_{p+1} & \operatorname{Re} \lambda_{p+1} & & & \\
& & & & \ddots & & \\
& & & & & \operatorname{Re} \lambda_{n-1} & -\operatorname{Im} \lambda_{n-1} \\
& & & & & \operatorname{Im} \lambda_{n-1} & \operatorname{Re} \lambda_{n-1}
\end{array}\right] .
$$

It is clear that the $J C F$ of $J$ is $D=\operatorname{diag}\left\{\lambda_{1}, \lambda_{2}, \ldots, \lambda_{n}\right\}$. Let $C=\sum_{i \in K} E_{i, i+1}$, $K=\{2, \ldots, n-2\}$ such that $D+C$ is the prescribed $J C F$. Let $S$ the matrix in (3.2). Then $S C S^{-1}$ has the form (3.3) and by $(2.2) B=S(J+\epsilon C) S^{-1}$ is a matrix in $\mathcal{C} \mathcal{S}_{\lambda_{1}}$ with spectrum $\Lambda$ and, by similarity, with the prescribed JCF. Now, let

$$
\mathbf{q}^{T}=\left(\sum_{i=2}^{n} \lambda_{i},-\lambda_{2}, \ldots,-\lambda_{p},-\operatorname{Re} \lambda_{p+1}, \ldots,-\operatorname{Re} \lambda_{n-1}\right)
$$


and $A=B+\mathbf{e q}^{T}$. Since $\left|\operatorname{Re} \lambda_{i}\right| \geq\left|\operatorname{Im} \lambda_{i}\right|$, the entries in columns $2,3, \ldots, n$ of $A$ are all nonnegative. In the first column, the entries in positions $(i, 1), i=2, \ldots p$, are of the form $\lambda_{1}-\lambda_{i}+\sum_{i=2}^{n} \lambda_{i}$ or they are of the form $\lambda_{1}-\lambda_{i}+\sum_{i=2}^{n} \lambda_{i}-\epsilon$. For the positions $(i, 1), i=p+1, \ldots, n$, the entries are of the form $\lambda_{1}-\operatorname{Re} \lambda_{p+j}-\operatorname{Im} \lambda_{p+j}+\sum_{i=2}^{n} \lambda_{i}$ or of the form $\lambda_{1}-\operatorname{Re} \lambda_{p+j}-\operatorname{Im} \lambda_{p+j}+\sum_{i=2}^{n} \lambda_{i}-\epsilon$. Since $\lambda_{1}+\sum_{i=2}^{n} \lambda_{i}>0,-\lambda_{i}>0$, $i=2, \ldots, p$, and $-\operatorname{Re} \lambda_{p+j}-\operatorname{Im} \lambda_{p+j} \geq 0, j=1, \ldots, n-p$, then the entries on the first column of $A$ are all nonnegative for $0<\epsilon \leq \min \left\{-\lambda_{2}, \lambda_{1}+\sum_{i=2}^{n} \lambda_{i}\right\}$. Thus, $A \in \mathcal{C S}_{\lambda_{1}}$ is nonnegative with spectrum $\Lambda$ and by Lemma 3.1 it has the prescribed elementary divisors. $\mathbf{L}$

Corollary 3.5. Let $\Lambda=\left\{\lambda_{1}, \ldots, \lambda_{n}\right\}$ with

$$
\lambda_{1}>\lambda_{2} \geq \cdots \geq \lambda_{p} \geq 0>\lambda_{p+1} \geq \cdots \geq \lambda_{n}, \quad 2 \leq p<n .
$$

If

$$
\lambda_{1} \geq \lambda_{2}-\sum_{j=p+1}^{n} \lambda_{j},
$$

when the nonnegative numbers $\lambda_{2}, \ldots, \lambda_{p}$ are all distinct or if

$$
\lambda_{1}>\lambda_{2}-\sum_{j=p+1}^{n} \lambda_{j}
$$

when some of the numbers $\lambda_{2}, \cdots, \lambda_{p}$ has algebraic multiplicity $m>1$, then there exists a nonnegative matrix $A \in \mathcal{C S}_{\lambda_{1}}$, with spectrum $\Lambda$ and prescribed elementary divisors $\left(\lambda-\lambda_{1}\right),\left(\lambda-\lambda_{2}\right)^{n_{2}}, \ldots,\left(\lambda-\lambda_{k}\right)^{n_{k}}, n_{2}+\cdots+n_{k}=n-1$.

Proof. Let $D=\operatorname{diag}\left\{\lambda_{1}, \lambda_{2}, \ldots, \lambda_{n}\right\}$ and let $S$ be the matrix in (3.2). Then by (2.2) $S D S^{-1} \in \mathcal{C S}_{\lambda_{1}}$. Let $C=\sum_{i \in K} E_{i, i+1}$, with $K \subset\{2, \ldots, n-1\}$ in such a way that $B=S D S^{-1}+\epsilon S C S^{-1}, \epsilon>0$, has $J C F \quad D+C(D+\epsilon C$ is similar to $D+C)$. Let

$$
q^{T}=(\sum_{j=p+1}^{n} \lambda_{i}, \underbrace{0, \ldots, 0}_{p-1 \text { times }},-\lambda_{p+1}, \ldots,-\lambda_{n}\} .
$$

Then the entries of $A=B+\mathbf{e q}^{T}$ in columns $2,3, \ldots, n$ are all nonnegative. The entries in positions $(i, 1), 2 \leq i \leq n$ on the first column of $A$ are of the form

$$
\lambda_{1}-\lambda_{i}+\sum_{j=p+1}^{n} \lambda_{j}, \quad i \in\{2, \ldots, n\},
$$

or they are of the form

$$
\lambda_{1}-\lambda_{i}-\epsilon+\sum_{j=p+1}^{n} \lambda_{j}, \quad i \in\{2, \ldots, n\} .
$$


Since

$$
\lambda_{1}-\lambda_{i}+\sum_{j=p+1}^{n} \lambda_{j} \geq \lambda_{1}-\lambda_{2}+\sum_{j=p+1}^{n} \lambda_{j} \geq 0, \quad i=2, \ldots, n,
$$

the second inequality being true from (3.5) and (3.6), then the entries of the form (3.7) are all nonnegative (whether the nonnegative numbers $\lambda_{2}, \ldots, \lambda_{p}$ are distinct or not). However, for the entries of the form (3.8) we have two cases:

i) If $\lambda_{2}, \ldots, \lambda_{p}$ are all distinct, then the entries of the form (3.8) appear only in certain positions $(i, 1)$, for $i=p+1, \ldots, n$. Since

$$
\lambda_{1}-\lambda_{i}+\sum_{j=p+1}^{n} \lambda_{j} \geq \lambda_{1}-\lambda_{p+1}+\sum_{j=p+1}^{n} \lambda_{j}>\lambda_{1}-\lambda_{2}+\sum_{j=p+1}^{n} \lambda_{j},
$$

$i=p+1, \ldots, n$, then from condition (3.5), we can choose

$0<\epsilon<\lambda_{1}-\lambda_{p+1}+\sum_{j=p+1}^{n} \lambda_{j}$ such that $\lambda_{1}-\lambda_{i}+\sum_{j=p+1}^{n} \lambda_{j}-\epsilon \geq 0$.

ii) If some of the nonnegative eigenvalues $\lambda_{2}, \ldots, \lambda_{p}$ are equal, then the entries of the form (3.8) can also appear in positions $(i, 1)$, for $i=2, \ldots, p$. In this case from (3.9)

$$
\lambda_{1}-\lambda_{i}+\sum_{j=p+1}^{n} \lambda_{j}-\epsilon \geq \lambda_{1}-\lambda_{2}+\sum_{j=p+1}^{n} \lambda_{j}-\epsilon \geq 0
$$

for

$$
0<\epsilon \leq \lambda_{1}-\lambda_{2}+\sum_{j=p+1}^{n} \lambda_{j}
$$

which is condition (3.6). Thus, $A \in \mathcal{C S}_{\lambda_{1}}$ is nonnegative with spectrum $\Lambda$ and the prescribed elementary divisors.

Observe that if $\lambda_{1} \geq-\sum_{j=p+1}^{n} \lambda_{j}$, then we may split out the set $\Lambda=\left\{\lambda_{1}, \ldots, \lambda_{n}\right\}$ into $\Lambda_{1}=\left\{\lambda_{1}, \lambda_{p+1}, \ldots, \lambda_{n}\right\}$ and $\Lambda_{2}=\left\{\lambda_{2}, \ldots, \lambda_{p}\right\}$, and to use Theorem 3.2 and Theorem 3.3 to obtain a nonnegative matrix $A=A_{1} \oplus A_{2}$ with spectrum $\Lambda=\Lambda_{1} \cup \Lambda_{2}$ and prescribed elementary divisors

$\left(\lambda-\lambda_{1}\right),\left(\lambda-\lambda_{2}\right)^{n_{2}}, \ldots,\left(\lambda-\lambda_{k}\right)^{n_{k}}, n_{2}+\cdots+n_{k}=n-1$, where $A_{1}$ and $A_{2}$ are nonnegative with spectrum $\Lambda_{1}$ and $\Lambda_{2}$ and $J(A)=J\left(A_{1}\right) \oplus J\left(A_{2}\right)$.

The following result gives a sufficient condition for the existence of a positive matrix with arbitrarily prescribed real elementary divisors $\left(\lambda-\lambda_{1}\right),\left(\lambda-\lambda_{2}\right)^{n_{2}}, \ldots,(\lambda-$ $\left.\lambda_{k}\right)^{n_{k}}, n_{2}+\cdots+n_{k}=n-1$. The proof is constructive, in the sense that we can always construct a solution matrix, and it combines results related to the nonnegative inverse 
eigenvalue problem ( [3], [15]) with results in Section 2. The result in [15] is recent and gives a symmetric version of a perturbation result, due to Rado and presented by Perfect in $([13], 1955)$. This symmetric version is used in [15] to obtain a new, more general, sufficient condition for the existence of a symmetric nonnegative matrix with prescribed spectrum. The result in [3] establishes that if $\Lambda=\left\{\lambda_{1}, \ldots, \lambda_{n}\right\}$ is the spectrum of a symmetric nonnegative matrix, with $\lambda_{1}$ being the Perron root, and $\epsilon>0$, then $\Lambda_{\epsilon}=\left\{\lambda_{1}+\epsilon, \lambda_{2}, \ldots, \lambda_{n}\right\}$ is the spectrum of a symmetric positive matrix.

TheOREm 3.6. Let $\Lambda=\left\{\lambda_{1}, \ldots, \lambda_{n}\right\}$ be a list of real numbers and let $\Lambda_{\delta}=\left\{\lambda_{1}-\delta, \lambda_{2}, \ldots, \lambda_{n}\right\}$ with $\lambda_{1}-\delta \geq \lambda_{2} \geq \cdots \geq \lambda_{n}$ and $\delta>0$. Suppose there exists:

i) A partition of $\Lambda_{\delta}$ into $r$ nonempty sublists

$$
\Lambda_{k}=\left\{\lambda_{k 1}, \lambda_{k 2}, \ldots, \lambda_{k p_{k}}\right\}, \lambda_{11}=\lambda_{1}-\delta, \lambda_{k 1} \geq 0 ; \lambda_{k 1} \geq \ldots \geq \lambda_{k p_{k}}
$$

such that $\Gamma_{k}=\left\{\omega_{k}, \lambda_{k 2}, \ldots, \lambda_{k p_{k}}\right\}$, where $0 \leq \omega_{k} \leq \lambda_{1}-\delta, k=1, \ldots, r$, is the spectrum of a $p_{k} \times p_{k}$ symmetric nonnegative matrix, and

ii) An $r \times r$ symmetric nonnegative matrix with eigenvalues $\lambda_{11}, \lambda_{21}, \ldots, \lambda_{r 1}$ and diagonal entries $\omega_{1}, \omega_{2}, \ldots, \omega_{r}$.

Then there exists an $n \times n$ positive matrix $A$ with spectrum $\Lambda$ and with any legitimately prescribed elementary divisors $\left(\lambda-\lambda_{1}\right),\left(\lambda-\lambda_{2}\right)^{n_{2}}, \ldots,\left(\lambda-\lambda_{k}\right)^{n_{k}}, n_{2}+\cdots+n_{k}=n-1$.

Proof. The list $\Lambda_{\delta}$ satisfies conditions of Theorem 3.1 in [15]. Then there exists an $n \times n$ symmetric nonnegative matrix $B_{\delta}$ with spectrum $\Lambda_{\delta}\left(B_{\delta}\right.$ can be computed from Theorem 3.1 in [15]). From the result in [3, Theorem 3.2] there exists an $n \times n$ symmetric positive matrix $B$ with spectrum $\Lambda$ ( $B$ can be computed from Theorem 3.2 in [3]). Since $B$ is symmetric, it is diagonalizable and then, from the result in $[9$, Theorem 1] there exists an $n \times n$ positive matrix $A$ with spectrum $\Lambda$ and with the prescribed elementary divisors $\left(\lambda-\lambda_{1}\right),\left(\lambda-\lambda_{2}\right)^{n_{2}}, \ldots,\left(\lambda-\lambda_{k}\right)^{n_{k}}, n_{2}+\cdots+n_{k}=n-1 . \square$

\section{Examples.}

EXAMPLE 4.1. Let $\Lambda=\{9,-2,-2,-1+i,-1+i,-1-i,-1-i\}$ be given. We shall construct a nonnegative matrix with spectrum $\Lambda$ and elementary divisors $(\lambda-9)$, $(\lambda+2)^{2},(\lambda+1-i)^{2},(\lambda+1+i)^{2}$. For $S$ defined in $(3.2), C=E_{23}+E_{56}$ and $\epsilon=1$, 
we have

$$
\begin{aligned}
& B^{\prime}=S\left[\begin{array}{ccccccc}
9 & 0 & 0 & 0 & 0 & 0 & 0 \\
0 & -2 & 0 & 0 & 0 & 0 & 0 \\
0 & 0 & -2 & 0 & 0 & 0 & 0 \\
0 & 0 & 0 & -1 & -1 & 0 & 0 \\
0 & 0 & 0 & 1 & -1 & 0 & 0 \\
0 & 0 & 0 & 0 & 0 & -1 & -1 \\
0 & 0 & 0 & 0 & 0 & 1 & -1
\end{array}\right] S^{-1} \\
&= {\left[\begin{array}{ccccccc}
9 & 0 & 0 & 0 & 0 & 0 & 0 \\
11 & -2 & 0 & 0 & 0 & 0 & 0 \\
11 & 0 & -2 & 0 & 0 & 0 & 0 \\
11 & 0 & 0 & -1 & -1 & 0 & 0 \\
9 & 0 & 0 & 1 & -1 & 0 & 0 \\
11 & 0 & 0 & 0 & 0 & -1 & -1 \\
9 & 0 & 0 & 0 & 0 & 1 & -1
\end{array}\right] }
\end{aligned}
$$

and

$$
B=B^{\prime}+S C S^{-1}=\left[\begin{array}{ccccccc}
9 & 0 & 0 & 0 & 0 & 0 & 0 \\
10 & -2 & 1 & 0 & 0 & 0 & 0 \\
11 & 0 & -2 & 0 & 0 & 0 & 0 \\
11 & 0 & 0 & -1 & -1 & 0 & 0 \\
8 & 0 & 0 & 1 & -1 & 1 & 0 \\
11 & 0 & 0 & 0 & 0 & -1 & -1 \\
9 & 0 & 0 & 0 & 0 & 1 & -1
\end{array}\right]
$$

Then

$$
A=B+\mathbf{e}(-8,2,2,1,1,1,1)=\left(\begin{array}{ccccccc}
1 & 2 & 2 & 1 & 1 & 1 & 1 \\
2 & 0 & 3 & 1 & 1 & 1 & 1 \\
3 & 2 & 0 & 1 & 1 & 1 & 1 \\
3 & 2 & 2 & 0 & 0 & 1 & 1 \\
0 & 2 & 2 & 2 & 0 & 2 & 1 \\
3 & 2 & 2 & 1 & 1 & 0 & 0 \\
1 & 2 & 2 & 1 & 1 & 2 & 0
\end{array}\right)
$$

has the prescribed elementary divisors. We may also obtain nonnegative matrices with any other legitimately prescribed elementary divisors.

Example 4.2. Let $(\lambda-7.5),(\lambda-5),(\lambda-1)^{2},(\lambda+4)^{2},(\lambda+6)$ be given. Then, let $\Lambda=\{7.5,5,1,1,-4,-4,-6\}$ and $\Gamma=\{7,5,1,1,-4,-4,-6\}$. In ([15], Theorem 3.1, Example 5.2) it was shown that $\Gamma$ is the spectrum of the symmetric nonnegative 
matrix

$$
B^{\prime}=\left(\begin{array}{ccccccc}
0 & 6 & \frac{\sqrt{10}}{10} & \frac{\sqrt{10}}{10} & \frac{\sqrt{10}}{10} & \frac{\sqrt{10}}{10} & \frac{\sqrt{10}}{10} \\
6 & 0 & \frac{\sqrt{10}}{10} & \frac{\sqrt{10}}{10} & \frac{\sqrt{10}}{10} & \frac{\sqrt{10}}{10} & \frac{\sqrt{10}}{10} \\
\frac{\sqrt{10}}{10} & \frac{\sqrt{10}}{10} & 0 & \frac{3+\sqrt{5}}{2} & \frac{3-\sqrt{5}}{2} & \frac{3-\sqrt{5}}{2} & \frac{3+\sqrt{5}}{2} \\
\frac{\sqrt{10}}{10} & \frac{\sqrt{10}}{10} & \frac{3+\sqrt{5}}{2} & 0 & \frac{3+\sqrt{5}}{2} & \frac{3-\sqrt{5}}{2} & \frac{3-\sqrt{5}}{2} \\
\frac{\sqrt{10}}{10} & \frac{\sqrt{10}}{10} & \frac{3-\sqrt{5}}{2} & \frac{3+\sqrt{5}}{2} & 0 & \frac{3+\sqrt{5}}{2} & \frac{3-\sqrt{5}}{2} \\
\frac{\sqrt{10}}{10} & \frac{\sqrt{10}}{10} & \frac{3-\sqrt{5}}{2} & \frac{3-\sqrt{5}}{2} & \frac{3+\sqrt{5}}{2} & 0 & \frac{3+\sqrt{5}}{2} \\
\frac{\sqrt{10}}{10} & \frac{\sqrt{10}}{10} & \frac{3+\sqrt{5}}{2} & \frac{3-\sqrt{5}}{2} & \frac{3-\sqrt{5}}{2} & \frac{3+\sqrt{5}}{2} & 0
\end{array}\right)
$$

Since $B^{\prime}$ is irreducible, then for any $\delta>0, B=B^{\prime}+\frac{\delta}{\mathbf{v}^{T} \mathbf{v}} \mathbf{v} \mathbf{v}^{T}$, where $\mathbf{v}$ is the Perron eigenvector of $B^{\prime}$, is a symmetric positive matrix with spectrum $\Lambda_{\delta}=\{7+\delta, 5,1,1,-4,-4,-6\}$ and with any legitimately prescribed elementary divisors. In particular, for $\delta=\frac{1}{2}$ we have

$$
B=\left(\begin{array}{ccccccc}
\frac{1}{8} & \frac{49}{8} & \frac{1}{8} \sqrt{10} & \frac{1}{8} \sqrt{10} & \frac{1}{8} \sqrt{10} & \frac{1}{8} \sqrt{10} & \frac{1}{8} \sqrt{10} \\
\frac{49}{8} & \frac{1}{8} & \frac{1}{8} \sqrt{10} & \frac{1}{8} \sqrt{10} & \frac{1}{8} \sqrt{10} & \frac{1}{8} \sqrt{10} & \frac{1}{8} \sqrt{10} \\
\frac{1}{8} \sqrt{10} & \frac{1}{8} \sqrt{10} & \frac{1}{20} & \frac{31+10 \sqrt{5}}{20} & \frac{31-10 \sqrt{5}}{20} & \frac{31-10 \sqrt{5}}{20} & \frac{31+10 \sqrt{5}}{20} \\
\frac{1}{8} \sqrt{10} & \frac{1}{8} \sqrt{10} & \frac{31+10 \sqrt{5}}{20} & \frac{1}{20} & \frac{31+10 \sqrt{5}}{20} & \frac{31-10 \sqrt{5}}{20} & \frac{31-10 \sqrt{5}}{20} \\
\frac{1}{8} \sqrt{10} & \frac{1}{8} \sqrt{10} & \frac{31-10 \sqrt{5}}{20} & \frac{31+10 \sqrt{5}}{20} & \frac{1}{20} & \frac{31+10 \sqrt{5}}{20} & \frac{31-10 \sqrt{5}}{20} \\
\frac{1}{8} \sqrt{10} & \frac{1}{8} \sqrt{10} & \frac{31-10 \sqrt{5}}{20} & \frac{31-10 \sqrt{5}}{20} & \frac{31+10 \sqrt{5}}{20} & \frac{1}{20} & \frac{31+10 \sqrt{5}}{20} \\
\frac{1}{8} \sqrt{10} & \frac{1}{8} \sqrt{10} & \frac{31+10 \sqrt{5}}{20} & \frac{31-10 \sqrt{5}}{20} & \frac{31-10 \sqrt{5}}{20} & \frac{31+10 \sqrt{5}}{20} & \frac{1}{20}
\end{array}\right)
$$

and $S^{-1} B S=\operatorname{diag}\{7.5,5,1,1,-4,-4,-6\}$, where the columns of $S$ are eigenvectors of $B$, is the $J C F$ of $B$. If we take $C=E_{3,4}+E_{5,6}$, then the positive matrix $A=$ $B+\frac{1}{20} S C S^{-1}=$

$$
=\left(\begin{array}{ccccccc}
\frac{1}{8} & \frac{49}{8} & \frac{1}{8} \sqrt{10} & \frac{1}{8} \sqrt{10} & \frac{1}{8} \sqrt{10} & \frac{1}{8} \sqrt{10} & \frac{1}{8} \sqrt{10} \\
\frac{49}{8} & \frac{1}{8} & \frac{1}{8} \sqrt{10} & \frac{1}{8} \sqrt{10} & \frac{1}{8} \sqrt{10} & \frac{1}{8} \sqrt{10} & \frac{1}{8} \sqrt{10} \\
\frac{1}{8} \sqrt{10} & \frac{1}{8} \sqrt{10} & \frac{9+\sqrt{5}}{200} & \frac{309+101 \sqrt{5}}{200} & \frac{157-49 \sqrt{5}}{100} & \frac{309-101 \sqrt{5}}{200} & \frac{309+97 \sqrt{5}}{200} \\
\frac{1}{8} \sqrt{10} & \frac{1}{8} \sqrt{10} & \frac{311+99 \sqrt{5}}{200} & \frac{11+\sqrt{5}}{200} & \frac{311+101 \sqrt{5}}{200} & \frac{311-101 \sqrt{5}}{200} & \frac{153-50 \sqrt{5}}{100} \\
\frac{1}{8} \sqrt{10} & \frac{1}{8} \sqrt{10} & \frac{77-25 \sqrt{5}}{50} & \frac{313+99 \sqrt{5}}{200} & \frac{4-\sqrt{5}}{25} & \frac{154+51 \sqrt{5}}{100} & \frac{313-99 \sqrt{5}}{200} \\
\frac{1}{8} \sqrt{10} & \frac{1}{8} \sqrt{10} & \frac{315-99 \sqrt{5}}{200} & \frac{305-103 \sqrt{5}}{200} & \frac{31+10 \sqrt{5}}{20} & \frac{1}{155+51 \sqrt{5}} \\
\frac{1}{8} \sqrt{10} & \frac{1}{8} \sqrt{10} & \frac{307+99 \sqrt{5}}{200} & \frac{156-49 \sqrt{5}}{100} & \frac{307-101 \sqrt{5}}{200} & \frac{78+25 \sqrt{5}}{50} & \frac{3}{50}
\end{array}\right)
$$

has the given elementary divisors $(\lambda-7.5),(\lambda-5),(\lambda-1)^{2},(\lambda+4)^{2},(\lambda+6)$. We may also obtain nonnegative matrices with the following elementary divisors:

$$
\begin{aligned}
& (\lambda-7.5),(\lambda-5),(\lambda-1),(\lambda-1),(\lambda+4),(\lambda+4),(\lambda+6) \\
& (\lambda-7.5),(\lambda-5),(\lambda-1)^{2},(\lambda+4),(\lambda+4),(\lambda+6) \\
& (\lambda-7.5),(\lambda-5),(\lambda-1),(\lambda-1),(\lambda+4)^{2},(\lambda+6) .
\end{aligned}
$$


Acknowledgment. We thank the referee for valuable comments that greatly improved the presentation of this paper.

\section{REFERENCES}

[1] A. Borobia, J. Moro, and R. Soto. Negativity compensation in the nonnegative inverse eigenvalue problem. Linear Algebra Appl., 393:73-89, 2004.

[2] A. Brauer. Limits for the characteristic roots of a matrix IV: Applications to stochastic matrices. Duke Math. J., 19:75-91, 1952.

[3] M. Fiedler. Eigenvalues of nonnegative symmetric matrices. Linear Algebra Appl., 9:119-142, 1974.

[4] C. R. Johnson. Row stochastic matrices similar to doubly stochastic matrices. Linear Multilinear Algebra, 10:113-130, 1981.

[5] T. J. Laffey and H. Šmigoc. Nonnegative realization of spectra having negative real parts. Linear Algebra Appl., 416:148-159, 2006.

[6] D. London. On diagonals of matrices doubly stochastically similar to a given matrix. Linear Algebra Appl., 244:305-340, 1996.

[7] F. Lubeck. On the computation of elementary divisors of integer matrices. J. Symbolic Comput., 33:57-65, 2002.

[8] C. Marijuán, M. Pisonero, and R. L. Soto. A map of sufficient conditions for the real nonnegative inverse eigenvalue problem. Linear Algebra Appl., 426:690-705, 2007.

[9] H. Minc. Inverse elementary divisor problem for nonnegative matrices. Proc. Amer. Math. Soc., 83:665-669, 1981.

[10] H. Minc. Inverse elementary divisor problem for doubly stochastic matrices. Linear Multilinear Algebra, 11:121-131, 1982.

[11] H. Minc. Nonnegative Matrices. John Wiley \& Sons, New York, 1988.

[12] H. Perfect. Methods of constructing certain stochastic matrices. Duke Math. J., 20:395-404, 1953.

[13] H. Perfect. Methods of constructing certain stochastic matrices II. Duke Math. J., 22:305-311, 1955.

[14] R. L. Soto. Existence and construction of nonnegative matrices with prescribed spectrum. Linear Algebra Appl., 369:169-184, 2003.

[15] R. L. Soto, O. Rojo, J. Moro, and A. Borobia. Symmetric nonnegative realization of spectra. Electron. J. Linear Algebra, 16:1-18, 2007.

[16] H. Suleimanova. Stochastic matrices with real characteristic numbers. Doklady Akad. Nauk SSSR, 66:343-345, 1949. 